\title{
SPECIAL PURPOSE COMPUTER FOR N-BODY PROBLEMS
}

\author{
D. SUGIMOTO, T. EBISUZAKI, T. ITO, J. MAKINO \\ Department of Earth Science and Astronomy \\ College of Arts and Sciences \\ University of Tokyo \\ 3-8-1 Komaba, Meguro, Tokyo 153 \\ Japan
}

\begin{abstract}
Backend processors for use in gravitational N-body problems were constructed. They are as cheap as a personal computer, yet their speed is comparable to a supercomputer. As an example we show some results of simulations which were done for the dynamical evolution of galaxies in a compact group.
\end{abstract}

\section{Introduction}

In the gravitational $\mathrm{N}$-body problems we integrate the Newtonian equation of motion,

$$
\begin{aligned}
& d^{2} \mathbf{r}_{i} / d t^{2}=\mathbf{F}_{i}=\sum_{j=1}^{N} \mathbf{f}_{i, j}, \quad \text { for } i=1,2, \ldots, N, \\
& \mathbf{f}_{i, j}=-G m_{j}\left(\mathbf{r}_{i}-\mathbf{r}_{j}\right) /\left[\left(\mathbf{r}_{i}-\mathbf{r}_{j}\right)^{2}+\varepsilon^{2}\right]^{3 / 2},
\end{aligned}
$$

where $\varepsilon$ is the softening parameter. This problem is divided into two parts, the force calculation and the time marching. For one-time step the former and the latter require $O\left(N^{2}\right)$ and $O(N)$ steps of calculations, respectively. The speed of calculations is greatly increased when the former is performed on specially designed hardware (Sugimoto et al. 1990).

We constructed such hardware in a pipeline, calling it GRAPE (GRAvity PipE). It is a backend processor connected to a workstation as a host machine. The host sends GRAPE the coordinates $\mathbf{r}_{j}$ (and the mass $m_{j}$ ) for $j=1,2, \ldots, N$. GRAPE calculates $\mathbf{f}_{i, j}$ and $\mathbf{F}_{i}$, and sends $\mathbf{F}_{i}$ back to the host for $i=1,2, \ldots, \mathrm{N}$. The amount of communications is only of $O(N)$ so that the communication bottleneck is avoided for large $N$. Though GRAPE is not programmable, the host is fully programmable and the GRAPE system can adapt itself to a variety of gravitational N-body problems.

\section{GRAPEs for collision-free and collisional systems}

Two types of GRAPE have been constructed, GRAPE-1 for collision-free and GRAPE-2 for collisional systems. Detailed architecture of GRAPE-1 is discussed by Ito et al. (1990). In GRAPE-1 each force $\mathbf{f}_{i, j}$ is calculated with 8-bit floating point format. The 3/2-th power, which appears in equation (2), is found from a table written in a read-only memory. As far as the collision-free evolution is concerned, it guarantees the necessary accuracy for the final $\mathbf{F}_{i}$, irrespective of the number of particles $N$ (Makino et al. 1990). A computed result with GRAPE-1 reproduced quite well the result obtained with a conventional machine of full word-length. In 
GRAPE-2, on the other hand, we use full $32 / 64$ bit formats since the effects of close encounters have to be accurately calculated in the collisional systems.

At present, GRAPE-1 is in operation with an 8-MHz clock and it calculates the forces at 240 Mflops-equivalent. Here, the word equivalent implies that it calculates as many forces as a 240 Mflops general purpose machine does. GRAPE-2 is still in tuning phase; it is operated with a 4$\mathrm{MHz}$ clock and calculates the forces at 40 Mflops. It has only one sub-pipeline, while GRAPE-1 has three sub-pipelines in parallel corresponding to the $x, y$, and $z$ components of the force.

We are now extending the GRAPEs as follows. For GRAPE- 2 we are implementing a forward extension of the pipeline which treats Aarseth's individual time-steps; for GRAPE-1 we are implementing logics to generate a list of neighbour particles, which will be useful in smoothed particle hydrodynamics, and also logics to calculate potentials which will be useful in analyzing the computed results. Both of these will be communicated back to the host on demand. GRAPE-1 will be assembled on one printed circuit board and eight of such boards will be connected in parallel. We are considering a further plan to put GRAPEs into one customized LSI chip and connect such chips massively in parallel.

\section{Merging of galaxies in a compact group.}

To show how GRAPE-1 performs we shall describe some simulation results. We calculated different cases for the merging of galaxies in a compact group, initially consisting of 10 galaxies.

In the first case, no dark matter is assumed to exist. Each galaxy is, in its initial state, represented, with 1024 mass points distributed in a Plummer model. Merging of component galaxies proceeds in about $4 t_{\mathrm{cr}}$, where $t_{\mathrm{cr}}$, is the initial crossing time of a galaxy over the size of the compact group. In the final stage of our simulation there remain three galaxies. Two of them are the merger remnants of 6 and 3 galaxies. The third is one of the original galaxies and is escaping from the system. This computation took about 100 hours at the machine speed of 120 Mflops.

In the second case, each galaxy is represented with 256 mass points. In addition, the dark matter is represented with 23040 points of the same mass which are distributed initially in a Plummer model extending over the size of the compact group. The gravitational interaction between all the 25600 mass points was fully calculated. The merging proceeds in $10 t_{\mathrm{cr}}$. Each galaxy suffers from dynamical friction, sinks gradually down to the central region of the dark matter, and therein, each merges with the other. The final result is a low density merger which is surrounded by an extended halo. This computation took about 200 hours at 240 Mflops.

The third case is also simulated; each of 10 galaxies is represented with 1024 mass points as in the first case, but the system is immersed in a fixed graviational potential which might represent the dark matter. Though the mass ratio of the dark matter to the galaxies is almost the same as in the second case, the result is very different because of the absence of dynamical friction. Mass points ejected from galaxies form an extended halo, but the core of each galaxy keeps its identity. The whole system looks like a multi-nucleus galaxy.

\section{References}

Ito, T., Makino, J., Ebisuzaki, T., Sugimoto, D. (1990), Computer Phys. Commun. in press. Makino, J., Ito, T., Ebisuzaki, T. (1990), Publ. Astron. Soc. Japan in press.

Sugimoto, D., Chikada, Y., Makino, J., Ito, T., Ebisuzaki, T., Umemura, M. (1990), Nature $345,33$. 